\title{
Correction to: Performance study of trial M-THGEM
}

\author{
Shao-Qing Deng ${ }^{1}$. Wen-Qi Yan ${ }^{2}$ - Dao-Jin Hong ${ }^{3} \cdot$ Bo-Xiang Yu ${ }^{2,4,5} \cdot$ Gao-Long Zhang ${ }^{1,6} \cdot$ Yu-Guang Xie ${ }^{2,4}$ \\ Published online: 16 January 2020 \\ (c) Institute of High Energy Physics, Chinese Academy of Sciences; Nuclear Electronics and Nuclear Detection Society 2020
}

\section{Correction to: \\ Radiation Detection Technology and Methods https://doi.org/10.1007/s41605-019-0150-y}

Upon request from the authors, the sequence of grant numbers are corrected as:

Nos. 11575221, 11975040, U1832130, 11475013.

The original article can be found online at https://doi.org/10.1007/ s41605-019-0150-y.

Gao-Long Zhang

zgl@buaa.edu.cn

Bo-Xiang Yu

yubx@ihep.ac.cn

1 School of Physics and Nuclear Energy Engineering, Beihang University, Beijing 100191, China

2 Institute of High Energy Physics, Chinese Academy of Sciences, Beijing 100049, China

3 University of Science and Technology of China, Hefei 230026, China

4 State Key Laboratory of Particle Detection and Electronics, Beijing 100049, China

5 University of Chinese Academy of Sciences, Beijing 100049, China

6 Beijing Advanced Innovation Center for Big Data-Based Precision Medicine, Beihang University, Beijing 100083 , China 